\title{
A BINOMIAL COEFFICIENT IDENTITY ASSOCIATED TO A CONJECTURE OF BEUKERS
}

\author{
Scott Ahlgren, Shalosh B. Ekhad, Ken Ono and Doron Zeilberger
}

AbstRACt. Using the WZ method, a binomial coefficient identity is proved. This identity is noteworthy since its truth is known to imply a conjecture of Beukers.

Received: January 28, 1998; Accepted: February 1, 1998

If $n$ is a positive integer, then let $A(n):=\sum_{k=0}^{n}\left(\begin{array}{l}n \\ k\end{array}\right)^{2}\left(\begin{array}{c}n+k \\ k\end{array}\right)^{2}$, and define integers $a(n)$ by

$$
\sum_{n=1}^{\infty} a(n) q^{n}:=q \prod_{n=1}^{\infty}\left(1-q^{2 n}\right)^{4}\left(1-q^{4 n}\right)^{4}=q-4 q^{3}-2 q^{5}+24 q^{7}-\cdots
$$

Beukers conjectured that if $p$ is an odd prime, then

$$
A\left(\frac{p-1}{2}\right) \equiv a(p) \quad\left(\bmod p^{2}\right)
$$

In $[\mathrm{A}-\mathrm{O}]$ it is shown that (1) is implied by the truth of the following identity.

Theorem. If $n$ is a positive integer, then

$$
\sum_{k=1}^{n} k\left(\begin{array}{l}
n \\
k
\end{array}\right)^{2}\left(\begin{array}{c}
n+k \\
k
\end{array}\right)^{2}\left\{\frac{1}{2 k}+\sum_{i=1}^{n+k} \frac{1}{i}+\sum_{i=1}^{n-k} \frac{1}{i}-2 \sum_{i=1}^{k} \frac{1}{i}\right\}=0 .
$$

Remark. This identity is easily verified using the WZ method, in a generalized form [Z] that applies when the summand is a hypergeometric term times a WZ potential function. It holds for all positive $n$, since it holds for $n=1,2,3$ (check!), and since the sequence defined by the sum satisfies a certain (homog.) third order linear recurrence equation. To find the recurrence, and its proof, download the Maple package EKHAD and the Maple program zeilWZP from http://www.math.temple.edu/ zeilberg . Calling the quantity inside the braces $c(n, k)$, compute the WZ pair $(F, G)$, where $F=c(n, k+1)-c(n, k)$ and $G=c(n+1, k)-c(n, k)$. Go into Maple, and type read zeilWZP; zeilWZP $(\mathrm{k} *(\mathrm{n}+\mathrm{k}) ! * * 2 / \mathrm{k} ! * * 4 /(\mathrm{n}-\mathrm{k}) ! * * 2, \mathrm{~F}, \mathrm{G}, \mathrm{k}, \mathrm{n}, \mathrm{N})$ :

\section{REFERENCES}

[A-O] S. Ahlgren and K. Ono, A Gaussian hypergeometric series evaluation and Apéry number congruences (in preparation).

[B] F. Beukers, Another congruence for Apéry numbers, J. Number Th. 25 (1987), 201-210.

[Z] D. Zeilberger, Closed Form (pun intended!), Contemporary Mathematics 143 (1993), 579-607.

Department of Mathematics, Penn State University, University Park, Pennsylvania 16802

E-mail address: ahlgren@math.psu.edu

Department of Mathematics, Temple University, Philadelphia, Pennsylvania 19122

E-mail address: ekhad@math.temple.edu; http://www.math.temple.edu/ $\mathrm{ekhad}$

Department of Mathematics, Penn State University, University Park, Pennsylvania 16802

E-mail address: ono@math.psu.edu; http://www.math.psu.edu/ono/

Department of Mathematics, Temple University, Philadelphia, Pennsylvania 19122

E-mail address: zeilberg@math.temple.edu; http://www.math.temple.edu/ zeilberg

The third author is supported by NSF grant DMS-9508976 and NSA grant MSPR-Y012. The last author is supported in part by the NSF.

Typeset by $\mathcal{A M}_{\mathcal{M}}$ - $\mathrm{T}_{\mathrm{E}} \mathrm{X}$ 